\title{
Dual role for the ADP/ATP translocator?
}

Arising from: J. E. Kokoszka et al. Nature 427, 461-464 (2004)

- he ADP/ATP translocator (or adenine nucleotide translocase; ANT) is thought to play a dual role: in the transport of ADP and ATP across the mitochondrial inner membrane and in the formation of the mitochondrial permeability-transition pore $(\mathrm{mtPTP})$, a nonspecific pore that is an important mediator of apoptosis (programmed cell death $)^{1-3}$. However, Kokoszka et al. ${ }^{4}$ have shown that mitochondria from livers of 'ANT-knockout' mice, in which the ANT has been genetically inactivated, still possess mtPTP activity. From this, the authors conclude that the ANT is a nonessential component of the mtPTP that may be dispensable for mtPTP-associated cell death $^{4}$. These results, which contradict previous evidence ${ }^{1,2}$ and cast doubt on a widely accepted model for the mtPTP (ref. 1), warrant scrutiny and call for a fundamental reappraisal of the role of the ANT in liver metabolism.

Nearly all essential metabolic pathways in the liver require an abundant supply of cytosolic ATP, with gluconeogenesis, urea synthesis and lipogenesis being the most demanding. Most of this ATP is supplied by mitochondrial oxidative phosphorylation and is exported to the cytosol by the ANT in exchange for incoming ADP — as illustrated, for example, by the inhibition of hepatic gluconeogenesis and urea synthesis by atractyloside (a specific inhibitor of the ANT) ${ }^{5}$. It is therefore surprising that mice without hepatic ANT can survive the loss of these essential metabolic pathways, let alone live normally for at least a year ${ }^{4}$.
What metabolic changes could account for this unexpected finding? The liver might switch to using much more glucose in order to generate ATP by glycolysis in the cytosol, but this would lead to massive production of lactic acid by the liver (and subsequent oxidation by red muscle and heart) that should be readily detected as an increase in blood lactate concentrations. And even this compensatory mechanism would not enable the liver to carry out gluconeogenesis, a task that would have to be taken over by the kidney. An alternative is that another member of the mitochondrial carrier family, such as the deoxynucleotide carrier or the ATP $-\mathrm{Mg} / \mathrm{P}_{\mathrm{i}}$ exchange carrier, might take over the role of the ANT ${ }^{3}$.

Or could it be that the mitochondria are not totally devoid of ANT activity, as Kokoszka et al. claim? Their indirect assay for this activity is not particularly sensitive, relying as it does on stimulation of the already enhanced rate of state- 3 respiration, although any residual activity must be relatively small.

The principal conclusion reached by the authors $^{4}$, that the ANT is not an essential component of the mtPTP, may also require qualification in the light of evidence that the ANT, together with cyclophilin D, does fulfil this role $\mathrm{e}^{1,2}$. Putting aside the possibility that there may be some residual ANT present, could there be an alternative explanation for the new observations?

The ANT is the most abundant member of a family of transporters that have a conserved structure ${ }^{3}$, and several members of this family will form channels under specific conditions ${ }^{1,6,7}$; it would be consistent with the authors' data if the ANT represented the normal target for cyclophilin-D binding that leads to mtPTP formation. However, in the absence of the ANT, another less abundant member of the carrier family might take its place, albeit, as observed by the authors, with less sensitivity to calcium and no sensitivity to ligands of the ANT.

Also, it has been proposed ${ }^{8}$ that the mtPTP may be formed from aggregates of denatured membrane proteins: these may normally be stabilized by a chaperone, but open into a pore when acted upon by cyclophilin D in the presence of calcium.

There is strong evidence that the mtPTP can be formed from purified ANT and cyclophilin $\mathrm{D}^{2,7,9}$, but this falls short of proof. More rigorous analysis of the reconstitution of the mtPTP from purified components should resolve this controversy. Such an analysis will be facilitated by the X-ray crystallographic structures of the ANT (ref. 10) and cyclophilin D(ref. 11).

\section{Andrew P. Halestrap}

Department of Biochemistry, University of Bristol, Bristol BS8 1TD, UK

e-mail:a.halestrap@bristol.ac.uk

doi:10.1038/nature02816

1. Halestrap, A. P. \& Brenner, C. Curr. Medicinal Chem. 10 1507-1525 (2003).

2. Halestrap, A. P., Doran, E., Gillespie, J. P. \& O’Toole, A. Biochem. Soc. Trans. 28, 170-177 (2000).

3. Palmieri, F. Pflugers Arch. 447, 689-709 (2004).

4. Kokoszka, J. E. et al. Nature 427, 461-464 (2004).

5. Stubbs, M., Vignais, P. V. \& Krebs, H. A. Biochem. J. 172, 333-342 (1978).

6. Dierks, T., Salentin, A. \& Krämer, R. Biochim. Biophys. Acta 1028, 281-288 (1990)

7. Brustovetsky, N., Tropschug, M., Heimpel, S., Heidkamper, D. \& Klingenberg, M. Biochemistry 41, 11804-11811 (2002).

8. He, L. H. \& Lemasters, J. J. FEBS Lett. 512, 1-7 (2002).

9. Crompton, M., Virji, S. \& Ward, J. M. Eur. J. Biochem. 258, 729-735 (1998).

10. Pebay Peyroula, E. et al. Nature 426, 39-44 (2003).

11. Waldmeier, P. C., Zimmermann, K., Qian, T., Tintelnot Blomley, M. \& Lemasters, J. J. Curr. Medicinal Chem. 10, 1485-1506 (2003). 